\title{
The Categorisation of Internet Memes - A Different Approach
}

\author{
Markus Baurecht*
}

\begin{abstract}
Memes have unwittingly been used in human communication for millennia already, but it is only recently that they have been defined as a concept. Just like in everyday life, memes also developed online. They are to be seen as part of the larger general definition of cultural memes as Dawkins defined them in his work "The Selfish Gene" (1976). In this paper a new approach for the categorisation of internet memes will be presented. In contrast to previous methods, it takes multiple characteristics, like the medium, the ratio of visual vs. text, the content and the type, into account and grasps the core idea the transmission of a unit of cultural information - behind memes.
\end{abstract}

\footnotetext{
* University of Klagenfurt; markusba@edu.aau.at
} 


\section{Introduction}

Pictures of cats being cute, pictures with quirky or relatable captions on them, or even the occasional 'Rick Roll'1 , are all examples of what is commonly referred to as memes. While these are certainly some good examples of online memes, they only present two specific types of memes and it is necessary to recognise them as such in order to create a framework for their internal categorisation. It is vital to recognise them as a type of a cultural meme and not as something separate that has nothing to do with the original definition of the cultural meme as Dawkins framed it in his original work (1976). To do that it is required to clearly define what cultural memes are and determine how internet/online memes fit into this space. Only then, it is possible to identify the weaknesses of current approaches on internet meme classification, learn from their inabilities, incorporate their successes and accordingly create an updated framework of categories that has the ability to reduce the number of blind spots to a minimum. Accordingly, it is vital to explore what a meme is and how they are used in the production of culture.

\subsection{Meme as a cultural concept}

Memes as evolutionary-biologist, Richard Dawkins observed them, are not a recent phenomenon. Humans have been using them subconsciously for millennia (Dawkins 1976). The term 'meme' is short for 'mimeme' which is derived from Ancient Greek and means 'imitated thing' (Rogers 2019). Imitation, as Dawkins describes it, is one of the central elements of memes (Dawkins 1976). More thoroughly, they are "an idea, a skill, a behaviour, a phrase or a particular fashion" (Rogers 2019) that carries a unit or package of cultural information and is transmitted when a person copies that unit or package through imitation (Dawkins 1976).

It is important to note that memes are exposed to the evolutionary process and should not be seen as something rigid and unchanging. Evolution happens in the form of random and involuntary mutations during the process of transmission (Dawkins 1976). Even natural selection plays a certain role in that process too, since it is regularly the case that the appearance of one meme causes the fading of another (Rogers 2019). This can be exemplified by greeting rituals of different groups. Group A might like to use handshakes, while group B might have a preference for fist-bumps. If members of the two groups meet, they would need to determine a common way of greeting one another. The groups could decide to either pick one of the already existing rituals, merge them as some sort of mutation or establish an entirely new one. Depending on the usefulness

\footnotetext{
1 A 'Rick Roll' describes the act of sending someone a link to the music video of Rick Astley's "Never Gonna Give You Up", disguised as a link to something that would actually be of interest to the recipient.
} 
that both groups ascribe to the common way of greeting they established, their internal ways of greeting might cease to exist in favour of the mutual one.

If observed further this is applicable to a diverse range of cultural practices like personal hygiene, working arrangements and even language. Some of the examples that might come to mind when thinking about this concept so broadly might even question the involuntary nature of meme mutations, but this aspect is still open to debate.

\subsection{Internet memes}

An easy way to define internet memes would be to say that they are inside jokes ${ }^{2}$ on the internet. They can be seen as pictures, videos, texts or a combination of these media, which share pieces of cultural information either individually or to an online audience (Rogers 2019).

As mentioned earlier, it is crucial to see online memes as a certain type of cultural meme, rather than something that is separate from cultural memes. They are a placeholder in communication and their purpose is to transmit larger pieces of information in a time-saving fashion. While at first glance observers might conclude that meaning is derived from the image that is being displayed, context is equally significant. What matters is what the image expresses, the underlying idea behind it and what the idea means to people.

This reproduces the factor of natural selection, which is present in the concept of cultural memes, because memes have to be recognisable in order to be useful. The usefulness of a meme determines its chance of survival. Borrowing from the greeting ritual example, a handshake has to be recognised as a greeting ritual in order to be useful. Similarly, the information that is transmitted through online memes has to be recognisable without further explanation in order to be useful. What this means is that the usefulness of an online meme is not necessarily dependent on which person or character is portrayed in the image or video, but rather whether people are able to recognise the information that is transmitted through them. Here some examples of frequently used images:

\footnotetext{
2 Jokes that only a select group of people can relate to.
} 


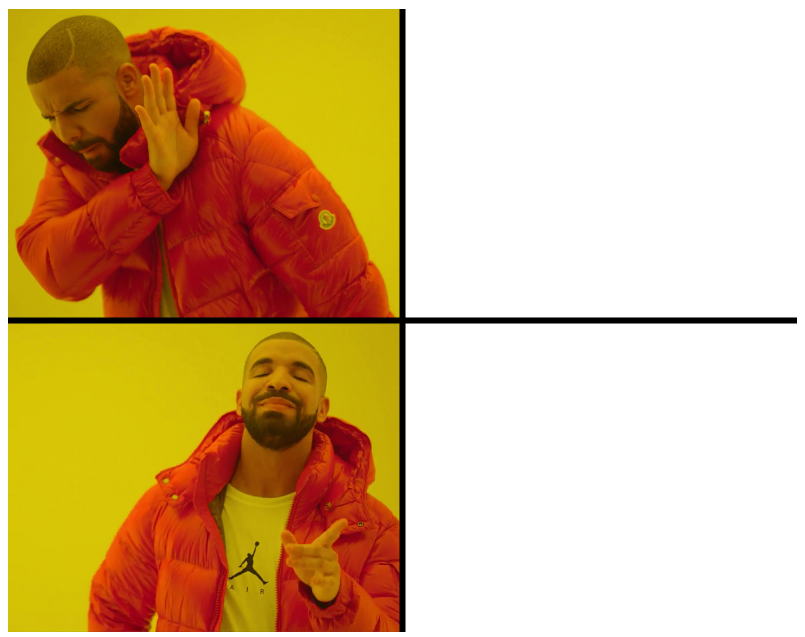

Figure 1: Drake Dislike/Like; expresses negative or positive emotions towards what is presented in the blank spaces

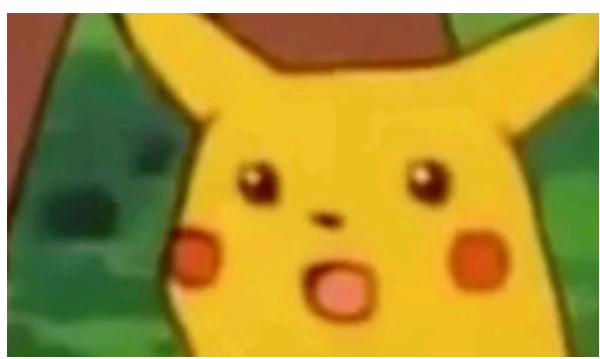

Figure 2: Surprised Pikachu; expresses the emotion of unexpected surprise

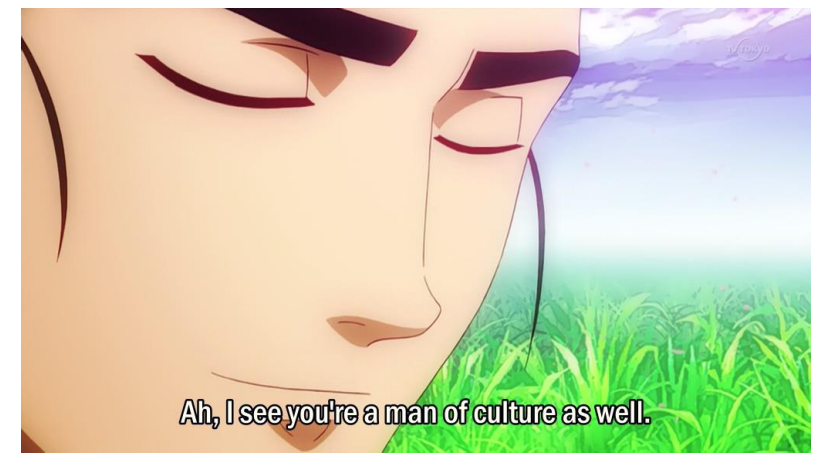

Figure 3: Ah I See You're A Man Of Culture As Well; expresses the admiration for certain knowledge/behavior and therefore recognition as a "cultured" person 


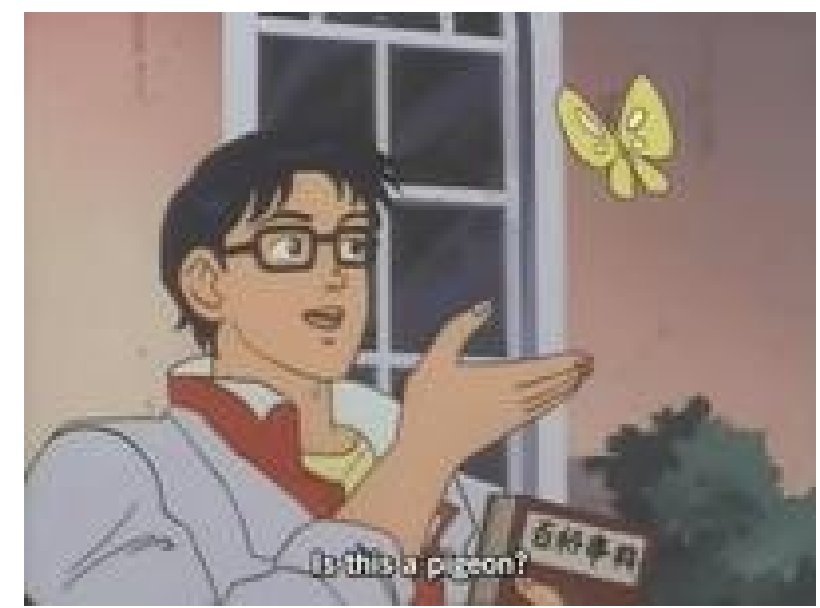

Figure 4: Is This A Pigeon?; expresses the inability of someone to recognize something obvious for what it is

It is not necessary for users to know who is being portrayed to understand what is trying to be communicated. The expression and gestures of people or subtitles are usually enough for them to be recognisable. In part this is the challenge of internet meme creation. It can be difficult to find a certain image or video which represents a specific emotion or situation perfectly. This can be observed by how subtle the difference between some templates with seemingly similar meaning can be.

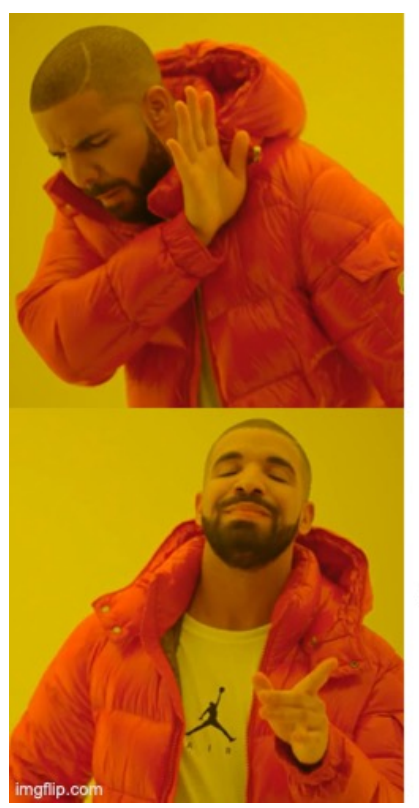

\title{
Sleep
}

\author{
Playing \\ Video Games \\ till 5am
}

Figure 5: Drake dislikes “Sleep”. Drake likes “Playing Viddeo Games till 5am”. 


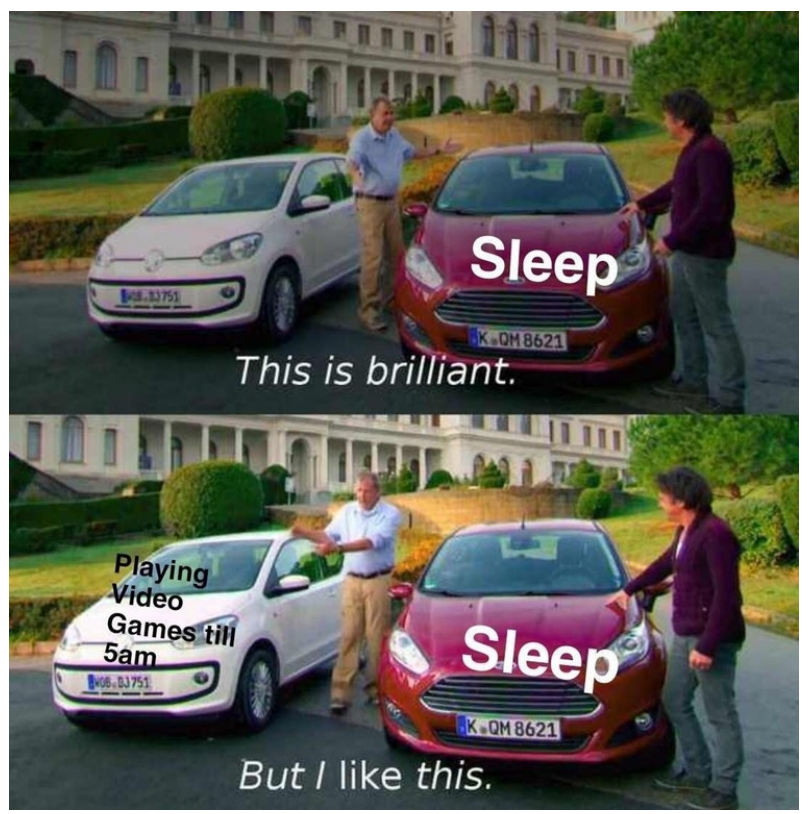

Figure 6: Jeremy recognises that „Sleep“ is brilliant, but he likes "Playing Video Games till 5am”.

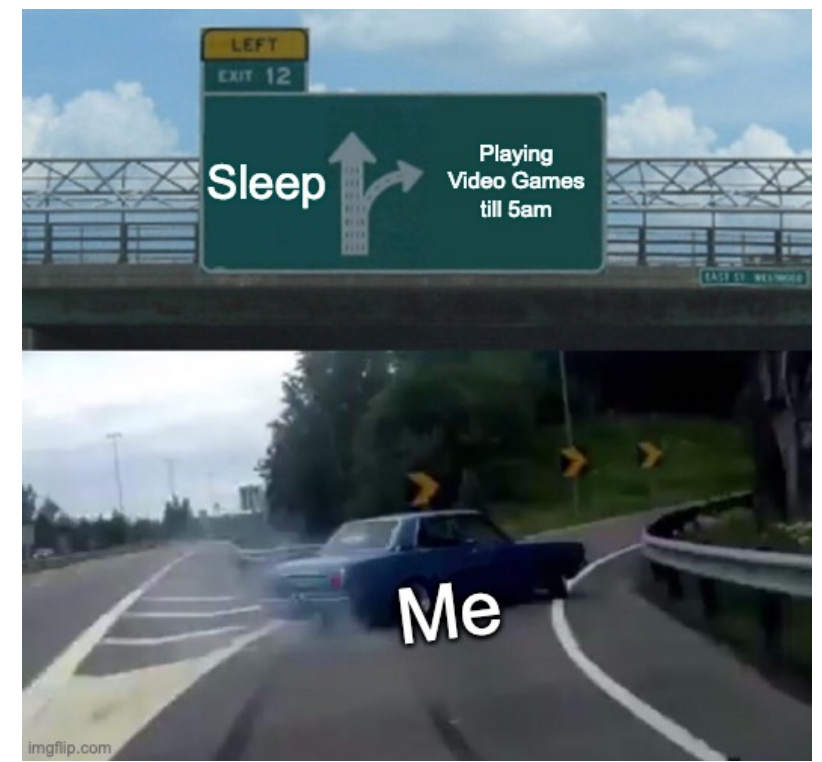

Figure 7: Taking a last minute decision to take the exit to "Playing Video Games till 5am" rather than going straight to "Sleep". 


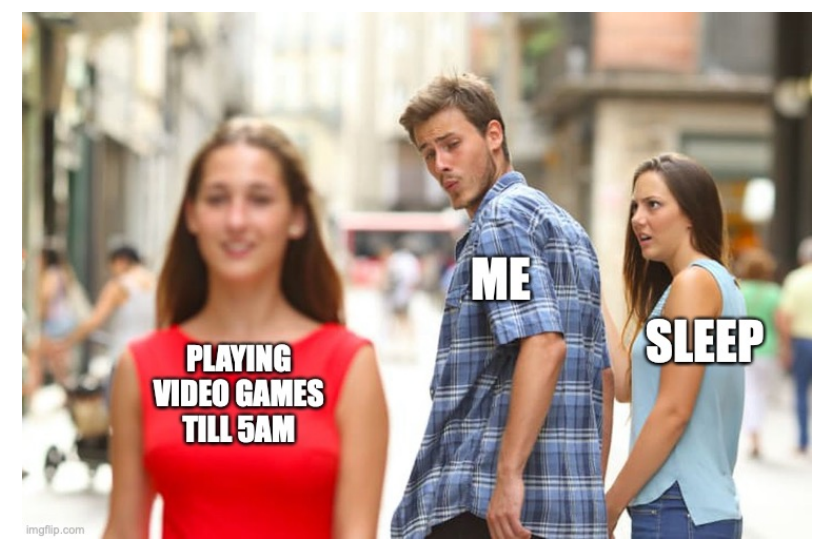

Figure 8: Although they already have "Sleep" they are more attracted to "Playing Video Games till 5am”.

These examples show the subtle differences how certain pictures can express different kinds of emotions. Figure 5 shows clear dislike for one thing, in that case "Sleep", while expressing that they like the other thing "Playing Video Games till 5am". In Figure 6 the difference is that "Sleep" is presented as something that is brilliant, but they still like "Playing Video Games till 5am”. In Figure 7 a last-minute decision between two things is presented. Lastly, Figure 8 proposes that they have "Sleep", but would rather have "Playing Video Games till 5am". Finally, this aspect of the meme being a placeholder for a much larger piece of cultural information that is transmitted, is key when evaluating existing frameworks of internet meme classification and creating new ones.

\section{Current Frameworks for Classifying Internet Memes}

Internet memes are not a recent phenomenon, since they can be traced back until the 1990s. One of the first memes is called 'Godwin's Law' and was created during a time in which the internet grew in popularity (King 2013). Research on them has been conducted since the start of the new millennium, with a first mentioning in Susan Blackmore's work 'The Meme Machine' (2000). Since then, only a few approaches on the classification of internet memes have been proposed so far. While all of them focus on some important characteristics that have to be taken into account, none of them are capable of grasping the essence of what online memes are actually about as part of cultural memes. The unit of cultural information that is being transmitted, has to be recognised. However, because they offer some vital considerations, by focussing on certain characteristics of online memes, they provide a critical foundation upon which a more 
complex framework can be built. Concretely, four different frameworks have been reviewed closely in the process of creating a new approach. It has to be mentioned that not all of them have their origin in scientific literature and some are taken from news articles or posts on reddit. This is due to the lack of existing research on internet memes. While some are of non-scientific nature, they provide still provide the basic information that is required to create a more complex framework. These frameworks might not have been named by their authors, but for the purpose of this article, they were named after which trait of online memes they focus on.

\subsection{Content Approach}

Beattie (2012) puts forward an outline of four categories: Phrasal Memes, Video Memes, Interactive Memes, Promotional Memes. The main issue here is that the proposed outline mixes the characteristics by which online memes are grouped into different categories. one of the categories checks via which medium memes are presented. The other three assess how users experience them and what the content is about, respectively. While this framework of mixed criteria might not seem useful for categorisation, it provides some crucial food for thought for the creation of something more complex. It makes sense to pay attention to the medium in which a meme is presented (like other approaches propose as well), but also the content of the information that is transferred through the meme is vital to be assessed. While all the different aspects that this framework captures have to be recognised by a more sophisticated approach, the essential lesson form it is to analyse the meme's content.

\subsection{Medium Approach}

Similarly, the communications firm We are Flint ${ }^{3}$ proposes a rather simple system with the categories 'Image (Macro) Meme', 'Photo Meme', 'Video Meme' and 'Word Meme' in their report 'Memes, Memes Everywhere' (2017). This structure was also presented by Maha Chaudhry in the article 'A Scientific Breakdown of Memes' (2017). While it captures the importance of the medium in which a meme transmits information, it fails to recognise certain aspects that Beattie's model (2012) already identifies. This approach erroneously recognises 'Image (Macro) Memes' and 'Photo Memes' as separate categories, whereas in reality 'Image (Macro) Memes' fall in the category of 'Photo Memes', since the medium with which 'Image (Macro) Memes' are created are usually pictures. Thus, a subcategory (Image (Macro) Memes), which is part of an umbrella category (Picture Memes), is presented as a separate category, which is not useful, since this

\footnotetext{
3 'We Are Flint' changed their company name to 'Cast from Clay', hence the reference with different URL.
} 
would create a mix of characteristics. In addition, the aspects of interaction and purpose that Beattie provides that are required to grasp the complexity of memes are not touched upon here. Further, the category of "Word Meme" needs to be expanded, since observations show that contemporary online memes also occur as complete sentences, tweets or paragraphs rather than as single words, hashtags or phrases.

\subsection{Creation Approach}

Reddit user $\mathrm{u} /$ chaogomu tries to capture a more practical side of memes in his post 'Meme classification' (2017) on the subreddit 'Meme Economy'. This method is focussed on the aspect of meme creation and examines how the medium was incorporated in the process of the construction of the finished product. This example distinguishes between the categories 'Pure Memes', 'Template Memes' and 'Variant Memes'. Pure Memes, as he describes, are certain media that are posted in an unaltered form, but still carry certain meaning through contextual knowledge which depends on the context they are presented in (e.g. 'Rick Roll'). Template Memes are those which can be found all over the internet, have a common usage and are usually created this way (e.g. Surprised Pikachu). Variant memes usually take more effort to create. They are usually a combination of different photos/videos, with the captions of different templates combined in order to create new meaning. As $\mathrm{u} / \mathrm{ch}$ hogomu puts it, they are "high risk low reward" (Chaogomu 2017), since a lot of creativity goes into their creation while most of them are generally not going to be recognised by a larger audience and will not move on into the phase of usage as a template. In this attempt, the medium and purpose might not be incorporated, but the important property of interaction is. As mentioned in the chapter on memes as a cultural concept, memes evolve and mutate in order to retain usefulness. While this is not the only feature, that a suitable framework for meme classification should pay attention to, it certainly should be taken into account.

\subsection{Contextual Approach}

Lastly, a valuable contribution was put forward by Arturo Deza and Devi Parikh (2015), who did research on image virality. Although their main focus was not on trying to classify memes, their framework of criteria for image virality provided some critical considerations. Their model contained - among others - the facets of 'intrinsic context', which refers to "visual content that is intrinsic to the pixels of the image" (Deza \& Parikh $2015,1821)$ and 'textual context', which refers to the non-visual "caption or title”(Deza \& Parikh 2015, 1821) of the meme. This approach dives deeper into the idea that additional meaning is conveyed by certain characteristics of internet memes, rather than just observing what can be seen. This idea of content and text contextuality also has 
to be present in a more complex framework. As mentioned in Beattie's approach, this underlines the importance of content analysis in internet memes. Even further, here it is suggested to deconstruct the meme and separate the information that is transmitted visually from the one transmitted via text. Generally, this would take away the intended meaning of the meme which is usually created in the combination of both. However, this way an analysis can be done that determines whether a meme relies more on the textual or on the intrinsic context in regards to transmission.

\section{An Updated Framework for Classifying Internet Memes}

In order to pay attention to all the defining characteristics of internet memes (content, medium, creation, textual and visual context), since they are responsible for the successful transmission of the unit of cultural information, four main aspects through which they have to be analysed were established:

- Medium of Transmission: determines what type of medium is used to transmit information (image/picture ${ }^{4}$, video, text, GIF $^{5}$, etc.)

- Format of Transmission: determines what the ratio between textual and visual transmission inside a meme is (text heavy, visual heavy, balanced)

- Content of Transmission: determines what kind of information is transmitted (Politics/News/Current Affairs, Pop Culture, Relatableness ${ }^{6}$, Meme Self Reference, Science, Multiple)

- Types of Transmission: determines which way of meme creation was used

As seen in other approaches it is useful to start by determining what kind of medium was used to create a meme. This is important because it has certain implications for how information is transmitted, since information can be transmitted and perceived differently, depending on if presented via picture or video and also is an important factor when considering the other defining characteristics of internet memes.

Next, to examine the format of transmission is vital in order to determine how information is distributed: mainly textually, mainly visually or balanced? The format provides information about how much contextual knowledge concerning an image or text is necessary for the successful transmission of information. The deciding factor is not

\footnotetext{
4 Both image and picture mentioned, since the meme could be drawn or painted.

5 Usually a short looped video without sound.

${ }^{6}$ Refers to content that viewers can relate to emotionally or on the basis of personal experience.
} 
necessarily how much space the text or visual medium inhabit, but rather how much information they carry.

Third, the content of the information that is being transmitted via the meme also has to be inspected. Since the image and text are often used as a placeholder, the actual content of the information that is transmitted can only be decoded through contextual knowledge. Usually the content can only be determined by viewing the meme as a whole and how the different parts that make up the meme are combined with one another.

Finally, a typology of memes provides formation concerning the structure and creation of online memes. Each type contains a certain blueprint for how certain pieces of a meme need to be arranged. The type additionally influences how information is transmitted.

- Image Macro: Classic structure of an image as background visual with an introductory top text and a bottom text as the punchline.

- Panel Meme: Usually consist of 4 or more panels. Can take different forms, most commonly like vs. dislike.

- Description Meme: Different parts inside an image metaphorically stand for something else, which is commonly determined by additional text.

- Screenshot Meme: Screenshot taken from a movie, TV show, video often with subtitles.

- Crossover Meme: Commonly used but different meme images combined to create new meaning.

- Meta Meme: Self-referential to meme culture and/or breaking the third wall. Defying the laws of meme creation (Each type needs to follow a certain structure in order to be recognisable. Meta memes do not follow these laws/structures, but gain popularity because of their originality).

Here some examples for every one of the different types: 

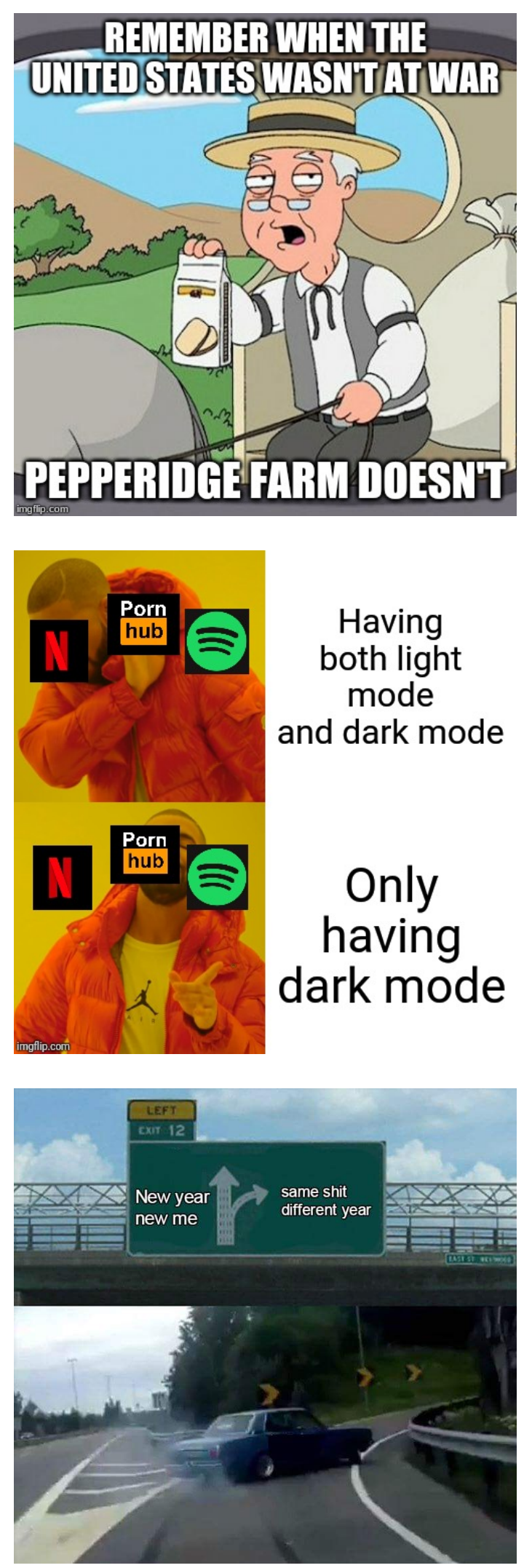

Figure 9: This meme was created using the so-called "Pepperidge Farm remembers template". Its origin is a commercial in the TV-show "Family Guy", where the person portrayed in the image, utters the catchphrase "Pepperidge Farm remembers". It is often used to refer to events in the past or typical situations in social life that used to happen, but do not anymore.

Figure 10: The template in Figure 10 contains screenshots of the music video to artist "Drake's" song "Hotline Bling”, clearly signalling to either dislike or like what is presented in the panels on the right.

Figure 11: In Figure 11, the descriptions that were added gave the road sign a new meaning. 
When the Jedi tell you that the Senate will decide your fate

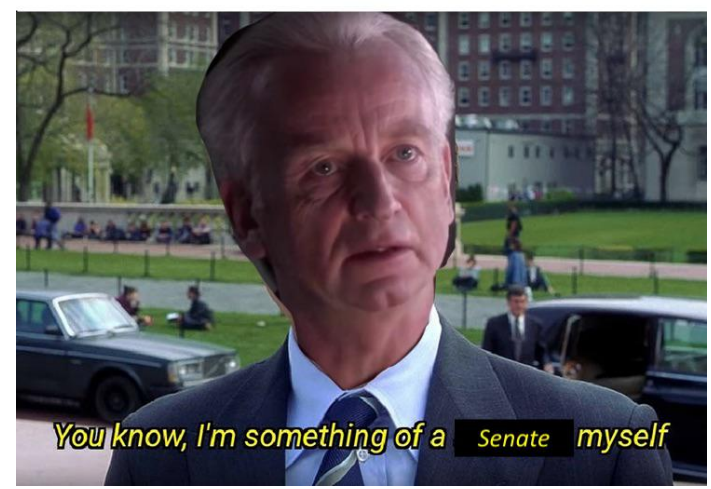

When someone reposts a meme that died in new and gets 10k upvotes
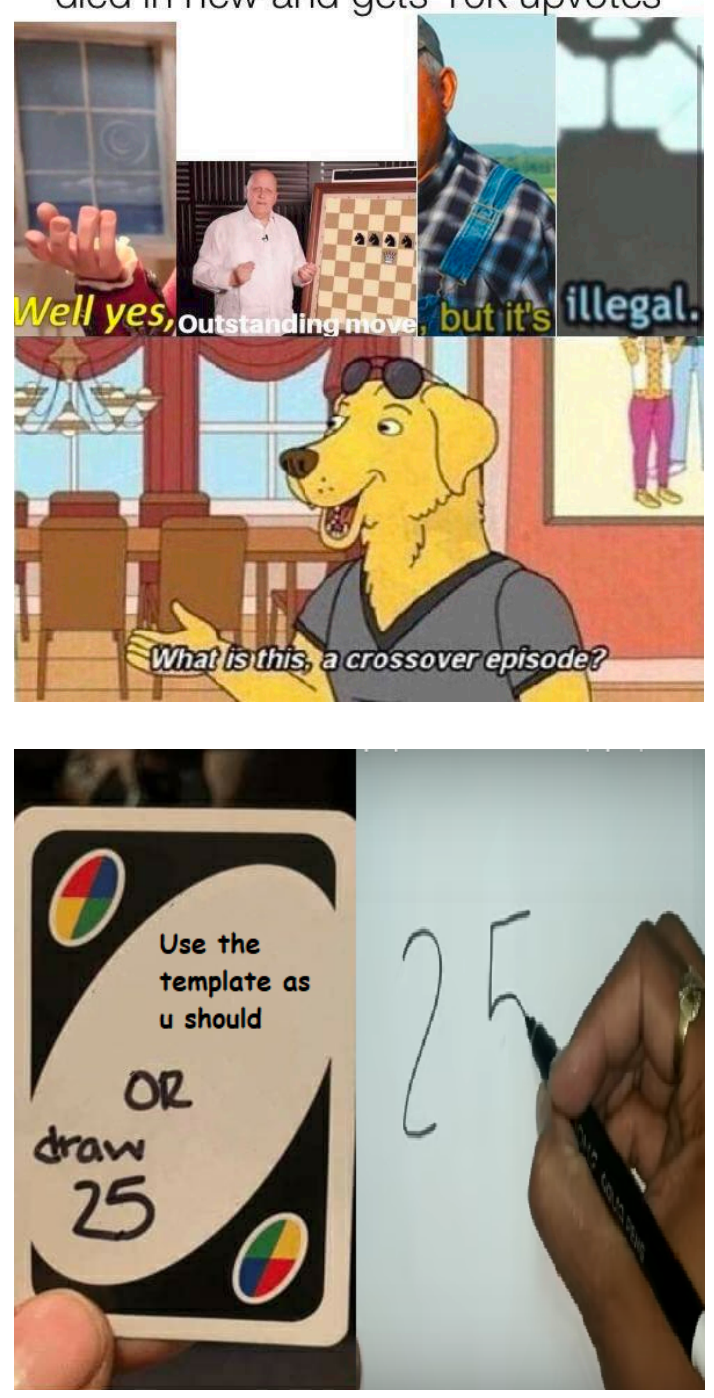

Figure 12: Figure 12 shows a variation of the "I'm Something of a Scientist Myself" template. This is an example where Photoshop was used to alter the text as well as the picture itself. In this version, it was combined with the "I am the Senate" template.

Figure 13: A combination of 5 different templates presents itself in Figure 13. The images of "Well Yes, But Actually No", "Outstanding Move", "But It's Honest Work" and "Wait, That's Illegal” were cut in such a way that their subtitles put together, formed a new sentence. Additionally, "What Is This, a Crossover Episode?” to serve as a punchline.

Figure 14: In Figure 14 the meme is a play on the usage of the template that was used to create it. Someone literally draws the number 25 instead of drawing 25 cards in the game "UNO", therefore the template is not used as it should be and because the template is not used correctly, someone has to "draw 25", which is taken literally. A circle of self-reference to the creation of a meme with this template emerges within the meme itself. 
It is important to note here is that with all these types, the combination of certain images with text, creates the meme. The image without the meme would usually not be enough. Similarly, the text without visual support would not be enough either. While it is possible for a meme to transmit information solely by visual or textual means, those examples are rather uncommon, but can still be covered by this framework.

\subsection{Application of the Updated Framework}

Using this framework, a survey on the website reddit.com and several of its subreddits ${ }^{7}$ has been conducted. Over the course of a seven-day period over 900 data points were collected. This data was gathered as follows:

Every day at 5 p.m. CET the subreddits r/all, r/memes, r/wholesomemes, r/dankmemes and $\mathrm{r} /$ historymemes were opened in the web browser. Each page was then sorted by daily popularity of the posts, in order to have the most popular posts of the day displayed. This is one feature why reddit.com was selected to conduct this research, since here it is possible to have the posts ordered without it being influenced by an algorithm that caters to personal preferences, like it is the case on Instagram or Facebook. ${ }^{8}$ r/all was selected since it presents all the posts that were posted on the whole website. The top 100 posts were surveyed and then determined how many of them could be considered to be memes. Afterwards, the eligible posts were analysed according to the new framework. The other pages were selected since they are the biggest subreddits which

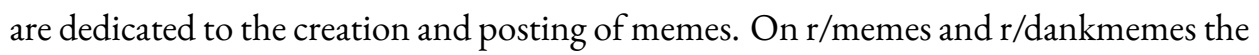
top 25 posts of the day were surveyed and analysed according to the new framework. On $\mathrm{r} /$ historymemes and $\mathrm{r} /$ wholesomemes this was done with only the top 20 and top 15 posts of the day, since the posts outside of this range usually do not gain the level of popularity of 1000 upvotes $^{9}$, which was determined to be adequate, considering the user base of the website and the individual subreddits.

\footnotetext{
7 A forum dedicated to a specific topic on the website Reddit.

8 Reddit allows to have a feed displayed that is only determined by how much upvotes certain posts have gotten and is not influenced by what Subreddits the user is subscribed to.

9 Upvotes and downvotes is the like and dislike function on Reddit.
} 


\begin{tabular}{lcccc}
\hline & Medium & Format & Content & Type \\
\hline r/all & $90 \%$ Image & $44 \%$ Balanced & $51 \%$ Pop Culture & $61 \%$ Screenshot \\
r/memes & $96 \%$ Image & $48 \%$ Balanced & $51 \%$ Pop Culture & $66 \%$ Screenshot \\
r/wholesomememes & $100 \%$ Image & $51 \%$ Balanced & $58 \%$ Relatable & $37 \%$ Panel \\
r/dankmemes & $96 \%$ Image & $54 \%$ Balanced & $49 \%$ Pop Culture & $65 \%$ Screenshot \\
r/HistoryMemes & $95 \%$ Image & $49 \%$ Balanced & $88 \%$ Pop Culture & $54 \%$ Screenshot \\
\hline
\end{tabular}

Table 1: Categorisation of memes posted on reddit.com

Table 1 shows that most of the data points collected fell in the category of images. The other columns also suggest that one category trumped the others (mentioned above in 3.) in most aspects. While that might be the case, it has to be noted that the other categories varied in percentage, depending on the subreddit analysed. Some contained more text-heavy than visual-heavy posts, or vice versa. Others varied in the different contents of the information that was transmitted through the memes. Even though 'Screenshot Memes' were largely constitute the most comprehensive type, 'Description Memes' and 'Panel Memes' were also well represented.

\section{Usefulness of the Updated Framework}

Judging from the results, this framework can be used as a functioning tool for the categorisation of memes. It found a large majority of posts to be created using images, which reflects the nature of reddit, how this website is used and how media is consumed there. In fact, the medium of posts depends on which subreddit is observed, so it comes as no surprise that in this instance, most posts were examined to be image-based.

Next, the format of posts was observed to be distributed more or less evenly, with slight variations depending on which subreddit was analysed. Usually the category of balanced distribution represented the majority of posts, which is likely connected to the visual-textual uniting nature of internet memes.

The aspects 'content of transmission' and 'type of transmission' were observed to not distribute the categories evenly. While this might likely be because of how posts are created and shared on this website, there is reason to believe that categories inside these areas were defined to broadly. Further research using this framework needs to be conducted on different websites to determine whether this is true and if the categories inside this factor of analysis need to be altered. However, even if it is determined that 
the currently used categories are not suitable, this does not conclude that the analysis of these characteristics is redundant. As mentioned earlier, they are crucial to grasp the essence of memes that was already mentioned by Dawkins when he observed the meme as a cultural concept, since they provide vital information about how the unit of cultural information is transmitted.

The updated framework sorts internet memes into different categories, while grasping the essence of the traditional definition of memes. It pays attention to all the different aspects that all the previous models suggested, incorporated in a single model. Beattie's "Content Approach" and the content aspect of Deza \& Parikh's "Contextual Approach" were covered in the new framework but how the content was analysed and assessed requires some further refinement. However, the aspect of "Framework of Transmission”, derived from Deza \& Parikh's approach, seemed to have functioned better, distributing the categories more evenly and providing more information about the relation between visual and textual transmission of information.

Further research could consider altering the aspect of "Medium of Transmission". It is necessary to differentiate memes along these lines (as We Are Flint \& Chaudhury suggested), since different media have different communicational properties. However, it seems that most of the information that is shared through memes, happens by images and pictures being used as a vessel for text, rather than GIFs or videos. This could be caused by the particularities of the website reddit, by difficulties encountered in meme creation with media other than images or pictures, or by completely different reasons. This also requires further inquiry.

Lastly, the categories inside the aspect of "Types of Transmission" derived from u/chaogomu's proposed approach still seem to lack the requirements to distribute properly and also do not properly grasp the creational facet of memes. The category of "Screenshot Meme" requires some alteration, since it is too close to being a subcategory of Image/Picture in the aspect of "Medium of Transmission" and does not quite grasp the creational component of memes like "Crossover Meme" and "Meta Meme" do.

While, the internal categorisation inside this framework needs further improvement, it provides a more sophisticated approach on meme classification that touches upon several different characteristics which are essential to the successful transmission of information through a meme. This approach does not yet serve as a finished product which can successfully be used to categorise all internet memes successfully but it contributes important considerations for future frameworks that previous ones lacked. In this model, all the different characteristics of internet memes that are necessary to grasp their essence are analysed and incorporated in the categorisation process. Existing models only focussed on one aspect, or mixed different ones together, like different aspects 
of content and medium. A useful approach requires to include all the facets of internet memes in order to grasp their complexity.

\section{References}

Beattie, Andrew. 2012. A Beginner's Guide to Internet Memes. Techopedia, January 2, 2012. https://www.techopedia.com/2/27179/internet/internet-meme/a-beginners-guide-to-internetmemes

Chaogomu. 2017. "Meme classification." r/MemeEconomy - Reddit.com. Accessed September 19, 2019. https://www.reddit.com/r/MemeEconomy/comments/5v7n5v/meme_classifi cation/.

Chaudhry, Maha. 2017. "A Scientific Breakdown of Memes.” Social Media Explorer, March 13, 2017. https://socialmediaexplorer.com/content-sections/tools-and-tips/a-scientific-break down-of-memes/.

Dawkins, Richard. 1976. The Selfish Gene. Oxford: University Press.

Deza, Arturo and Parikh, Devi. 2015. "Understanding Image Virality." IEEE Conference on Computer Vision and Pattern Recognition (CVPR) 2015. Accessed September 19, 2019. https: //arxiv.org/pdf/1503.02318.pdf.

King, Soraya. 2013. Mike Godwin's Law. From counter-memes to countering the FBI. The Guardian, October 29, 2013. https://www.theguardian.com/culture/2013/oct/29/mike-go dwin-nazi-analogies-meme-update

Rogers, Kara. 2019. “Meme. Cultural Concept.” Encyclopaedia Britannica, March 5, 2020. https://www.britannica.com/topic/meme

We Are Flint. 2017. "Memes, Memes Everywhere. An Introduction to Internet Memes." Cast From Clay. Accessed March 7, 2020. https://castfromclay.co.uk/full-report-meme-researchmemes-everywhere.

\section{Figures}

Figure 1: https://i.redd.it/4wmp5smh0ld41.jpg

Figure 2: https://i.kym-cdn.com/entries/icons/original/000/027/475/Screen_Shot_2018-10-25

_at_11.02.15_AM.png

Figure 3: https://i.kym-cdn.com/photos/images/original/001/236/841/075.jpg

Figure 4: https://i.kym-cdn.com/photos/images/masonry/000/911/850/e0a.jpg

Figure 5: https://imgflip.com/memegenerator/Drake-Hotline-Bling

Figure 6: https://i.kym-cdn.com/photos/images/original/001/366/527/562.jpg

Figure 7: https://imgflip.com/memegenerator/Left-Exit-12-Off-Ramp

Figure 8: https://imgllip.com/memegenerator/Distracted-Boyfriend

Figure 9: https://www.reddit.com/r/AdviceAnimals/comments/ekxgp1/this_perpetual_state_of_war/.

Figure 10: https://www.reddit.com/r/dankmemes/comments/elo9y1/dark_mode_superior/.

Figure 11: https://i.kym-cdn.com/photos/images/original/001/329/053/9ea.jpg. 
Figure 12: https://www.reddit.com/r/PrequelMemes/comments/em22ep/scientistsenate_same_thing/. Figure 13: https://www.reddit.com/r/memes/comments/aulta0/adderal_crossover_meme/.

Figure 14: https://www.reddit.com/r/dankmemes/comments/elxe80/oc_maymay/. 